\title{
$>$ Validation of the Dutch version of the Functional Recovery Index
}

\section{Background}

Traditionally, major complications and unanticipated (re)admission rates were used to assess outcome after day surgery. However, in view of the relative absence of major complications the quality of recovery (QOR) should be considered one of the principal endpoints after day surgery (Stessel 2015 Medicine). The English version of the Functional Recovery Index (FRI) is a validated tool developed to assess post discharge quality of recovery after ambulatory surgery (Wong 2009 anesthesiology). The aim of this study was to validate a Dutch version of the FRI.

\section{Methods}

We followed a three stage procedure. The first step was the forward translation of the English version of the FRI into Dutch. This was done by a bilingual person, a native speaker of the target language (Dutch). The second step was a separate back translation. This was performed by a bilingual translator who is a native speaker of the source language (English). The translations coincided. In step three a pilot with the Dutch version of the FRI was performed in a sample of 60 patients undergoing ambulatory surgery. Before surgery and one day after surgery, the FRI was applied and patients were asked to reflect on the comprehensibility of the questionnaire (numerical rating scale $0-10$ ) and asked for additional suggestions. Furthermore, cross-cultural validity was tested using confirmatory factor analysis on the data obtained one day after and before surgery.

1. Wong J, Tong D, De Silva Y, Abrishami A, Chung F. Development of the functional recovery index for ambulatory surgery and anesthesia. Anesthesiology. 2009;110(3):596-602. doi:10.1097/ALN.0b013e318197al6d.

2. Stessel B, Fiddelers AA, Joosten EA, Hoofwijk DMN, Gramke H-F, Buhre WFFA. Prevalence and Predictors of Quality of Recovery at Home After Day Surgery. Medicine (Baltimore). 2015;94(39):e1553. doi:10.1097/MD.0000000000001553.

\section{Results}

Only 6 patients scored an NRS $<8$ on the comprehensibility item. Mean NRS score on this item was 9.08 (SD 2.04). The results of the confirmatory factor analysis show that a model that assumes the three subscales as proposed by Wong et al. would fit the data better than a model with only one general recovery scale. The three subscales are a social related recovery scale (items 1-7), a lower limb recovery scale (items 8-11) and a physical recovery scale (items 12-14). Further improvement was found when allowing the factors to be correlated following their equivalence in measuring recovery. All items loaded on their respective subscales with loadings between 0.63 and 0.92 . The resulting subscales showed a high internal consistency as indicated by a Cronbach a (social = 0.91 (7 items), lower limb =0.91 (4 items), physical = 0.89 (3 items)). A similar Cronbach a was established for the same three subscales using the data obtained before surgery.

\section{Internal consistency}

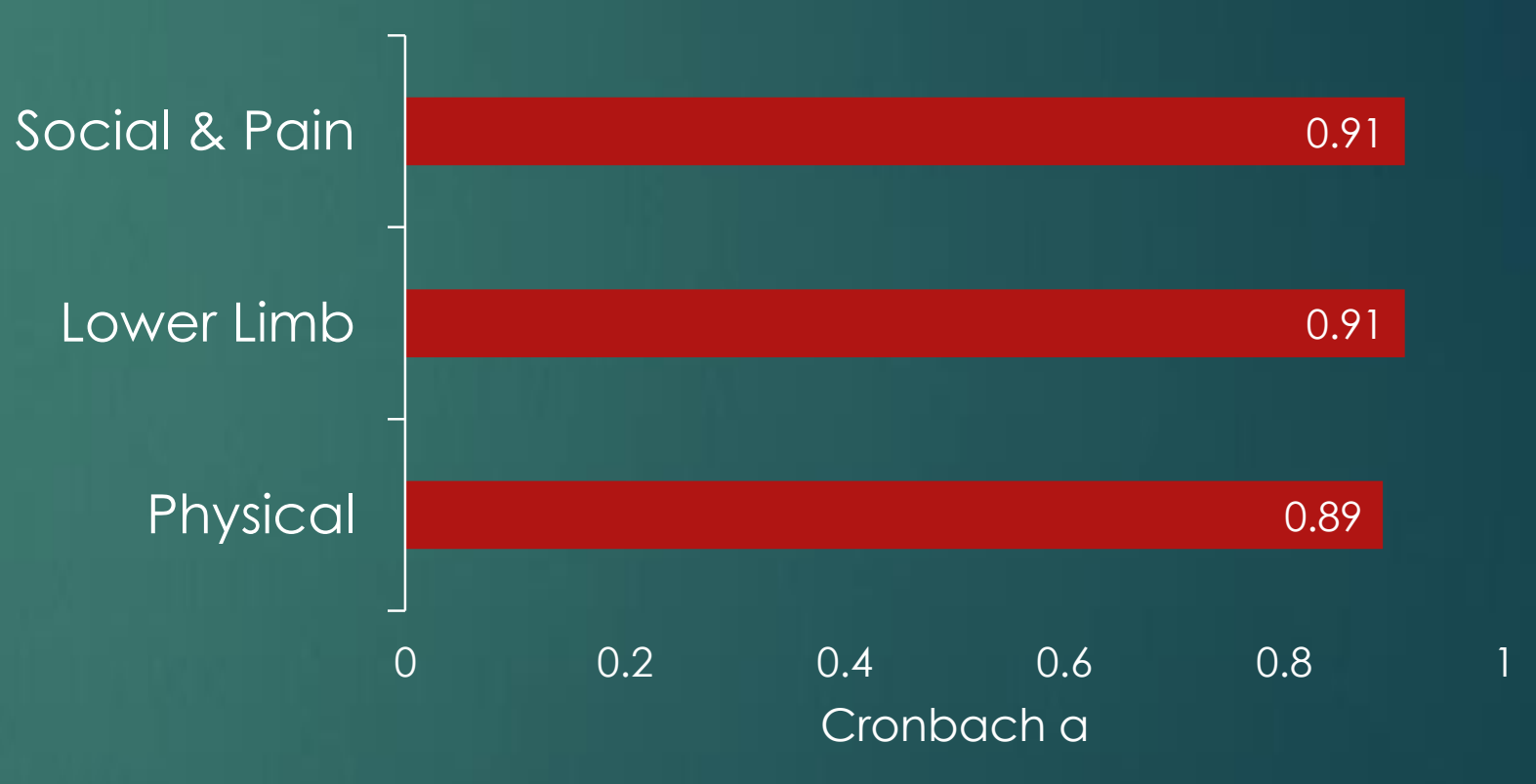

\section{Conclusion}

We can conclude that the study population considered the Dutch version of the FRI highly understandable. Confirmatory factor analysis showed that the three factor model, as proposed by Wong et al., also better fits the data of our study population than a one factor model.

Therefore, We state that our Dutch version of the FRI is a valid tool to asses recovery after ambulatory surgery in a Dutch-speaking population. 